\title{
Pattern Recognition on Vehicle Number Plates Using a Fast Match Algorithm
}

\author{
Cahaya Jatmoko*1, Durat Sinaga ${ }^{2}$ \\ 1,2Dian Nuswantoro Usiniversity, Semarang, Indonesia 50131 \\ E-mail : cahayajatmoko@dsn.dinus.ac.id ${ }^{* 1}$, dauratsinaga@dsn.dinus.ac.id ${ }^{2}$ \\ *Corresponding author
}

\author{
Edi Sugiarto ${ }^{3}$, Nur Rokhman ${ }^{2}$, Heru Lestiawan ${ }^{3}$ \\ 3,4,5 Dian Nuswantoro Usiniversity, Semarang, Indonesia 50131 \\ E-mail : edi.sugiarto@dsn.dinus.ac.id ${ }^{3}$,nurrokhman@dsn.dinus.ac.id ${ }^{4}$, \\ heru.lestiawan@dsn.dinus.ac.id ${ }^{5}$
}

\begin{abstract}
Computer Vision was the fast developing apps in the world, it is make people make a lot of new algorithm. Before we can use in out app, we need to test the algorithm to make sure how effective and optimal the algorithm to solve every case we given. A lot of traffic system has implemented computer vision, they need fast and can work in every condition, because every vehicle who pass needs to be recognized. In this research Fast Match algorithm was chosen because they can solve some test and make a lot of image have a similarity with the template. It makes accuracy of the data can be achieved with this algorithm. For example on of the sample was have a SAD point for 0.5 and Overlap Error for 0.5 and can run in standard computer just for a couple second. It makes the template and the original image has a little similarity.
\end{abstract}

Keywords - Computer Vision, Fast Match, Pattern Recognition, Digital Image

\section{INTRODUCTION}

One of the requirements for a vehicle to be officially driven in Indonesia is to have a TNKB (motorized vehicle number sign) or better known as a number plate. Each number plate has the same physical characteristics [1], namely for public vehicles that are black, made of iron and have characters and numbers printed uniquely and a black background [2]. With the aim of being a sign or characteristic, that the vehicle has an official letter and has been registered with the police. The introduction of vehicle numbers [3]-[5] is an interesting topic and is one of the main things in a system that requires vehicle number data such as parking systems, toll systems, and even traffic systems in the event of traffic violations [1], [6]. By keeping up with the times, it is hoped that everything that exists can be done automatically, so an application is created that can recognize a number plate through the entered image to retrieve the data [7]-[9].

Automation is one of the important elements in today's informatics era, with the emergence of various kinds of technology causing a very fast flow of information. Therefore, there are benefits such as the ease with which a person can take photos in everyday life. Then came an image processing technique that could automatically recognize the shape, color and character of an object [8]. Image recognition technique is a series of processes consisting of 
several methods. With the aim that an image can produce information by utilizing a variety of forms, features, colors, with the condition that it must be explicit in order to make a decision.

Image recognition or Image Recognition uses several steps before the image can be analyzed. One of them is making templates manually and automatically only through input images from the real world, that are taken using a cellphone camera. One of the algorithms used is the Fast Match Algorithm, where the fast match algorithm is an algorithm invented by [10]. It is an addition to the Template Matching algorithm by providing a 2D transformation of the input image. The Matching template uses the template as a reference in adjusting the parts of the image that need to be analyzed [11]. This method has a fairly short travel time in recognizing license plate images [12]. However, apart from a fairly short travel time, this algorithm has a weakness in the noise level in the image so that filtering is needed in this algorithm [5]. So, to cover the weaknesses of the Template Matching algorithm, an algorithm called Fast Match: Affine Template Matching was made, an algorithm proposed by [10] where this algorithm was created to suppress the SAD (Sum Of Difference) error value in measurement by taking a small part of the pixels in the input image. This algorithm has a weakness for images that have a high texture.

By taking the exposure from the background regarding image recognition with the algorithm used is the Fast Match Algorithm. The algorithm is chosen as an algorithm for image recognition on the condition that the motorized vehicle displays part of the number plate. In writing this study, the authors used some of the previous literature as a reference in this research. According to [5] there is still the need for exploration of this algorithm to make this algorithm very interesting, it is stated that improvisation or filtering to reduce the noise level can make this algorithm work much better, so it is hoped that in future studies not only 2D images but images in the form of videos can be used in real time. The next literature is an idea [13] saying that because the availability of training data makes the feature extraction process shorter in calculating the correlation value with training data, the main method used in this algorithm is convolution which is a step to get features from imagery. Of the 20 images that have been trained has an accuracy rate of $85 \%$. The position of the letters in the image can affect the weight value, from existing research. The higher the value of the Weight Value affects the success or failure of the algorithm in detecting images. Next is research from [14], he provides improvements to the template matching algorithm, namely by using gradient search assistance by changing the shape of the tempalate from a square to a round shape and then assisted by using Polar Coordinates as an effort to speed up the detection process quickly. From this research, it can be concluded that there is a time difference between the conventional template matching algorithm and the template matching algorithm that has been given 10 seconds gradient searching.

According to [15], the research he did was to change a 2D image into a $1 \mathrm{D}$ image by totaling the intensity value of the image, both horizontally and vertically. Then the second stage is to detect a template matching algorithm image on 1D images, then end with a decision making based on the similarity value function. Adding Gausian Noise affects decision making, resulting in a result that is $92.1 \%$ more accurate than $32 \mathrm{D}$ algorithms compared to NCC, SAD \& CTF. The last and which will be implemented in this study is a proprietary research [10] which is about the use of the Fast Match Algorithm in recognizing images. This algorithm is a development of a template matching algorithm with limitations in the form of unreadable images when given high noise, so that this algorithm is intended to reduce the SAD (sum of absolute difference) value and the overlap error value.

Based on literature research,here we wants to propose the Application of the Fast Match Algorithm in the Introduction of Number Plate Patterns on Motor Vehicles, to try to what extent this algorithm can work, images with different conditions will greatly affect the 
performance of this algorithm. The result will be the value of SAD, Overlap Error and Display template as well as the image and the detected image area. This research has been conducted by Matlab.

\section{RESEARCH METHOD}

\subsection{Data Collection}

In this study, the number of license plate images was 50 in unequal resolutions and then given the conditions, normal, blur and low light as shown in Figure 1.
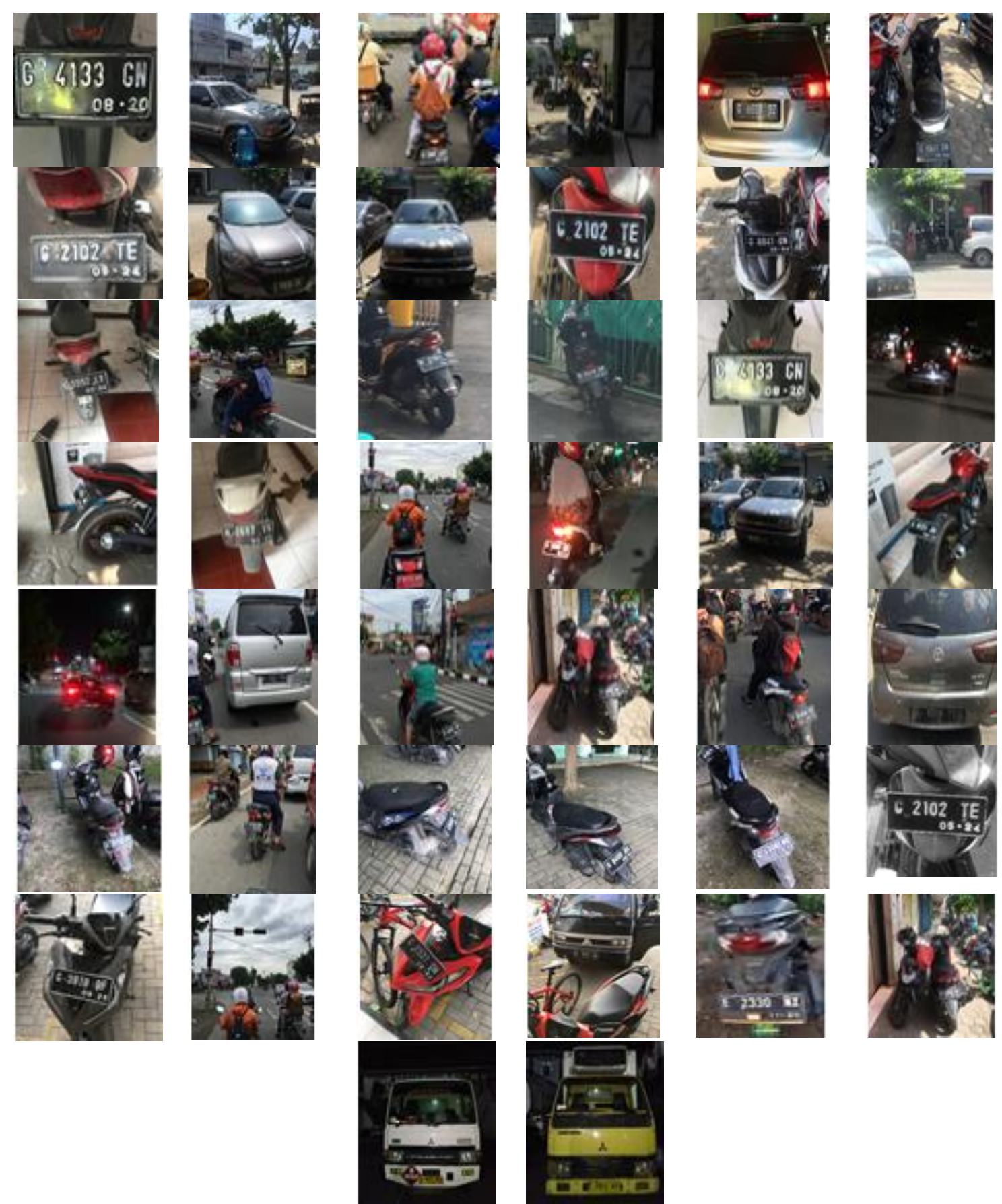

Figure 1. Data Collection for Image Recognition 


\subsection{Fast Match}

Fast Match is an updating algorithm of template matching where the use of image smoothness density is used as a $2 \mathrm{D}$ transformation of the image. This algorithm suppresses the SAD (Sum of Absolute Difference) [16] value and the Overlap Error value. The idea sparked by [10] is to take a sample from affinne transformation, then evaluate each transformation of the sample that has been taken, then return the sample to the closest distance to the template through the illustrations as in Figure 1.

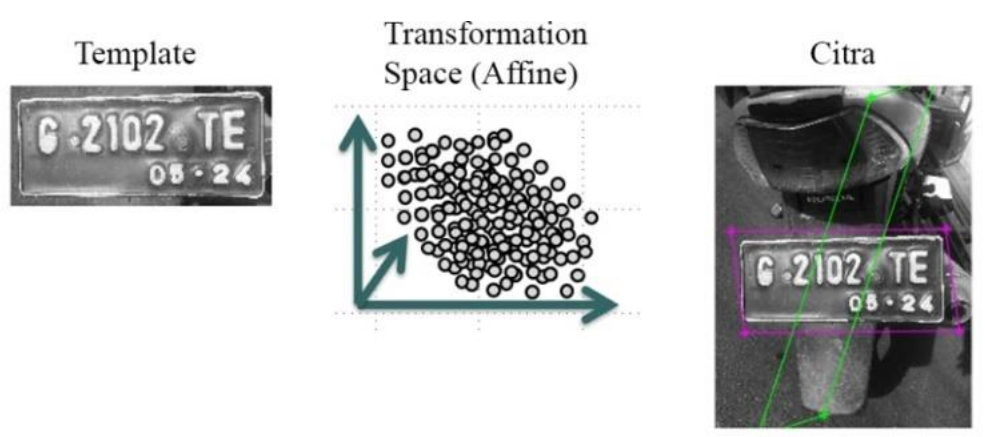

Figure 2. Illustration of Fast Match

\subsection{Research Stages}

The tool used to analyze this algorithm is Matlab, then the input and output will be analyzed with existing conditions.

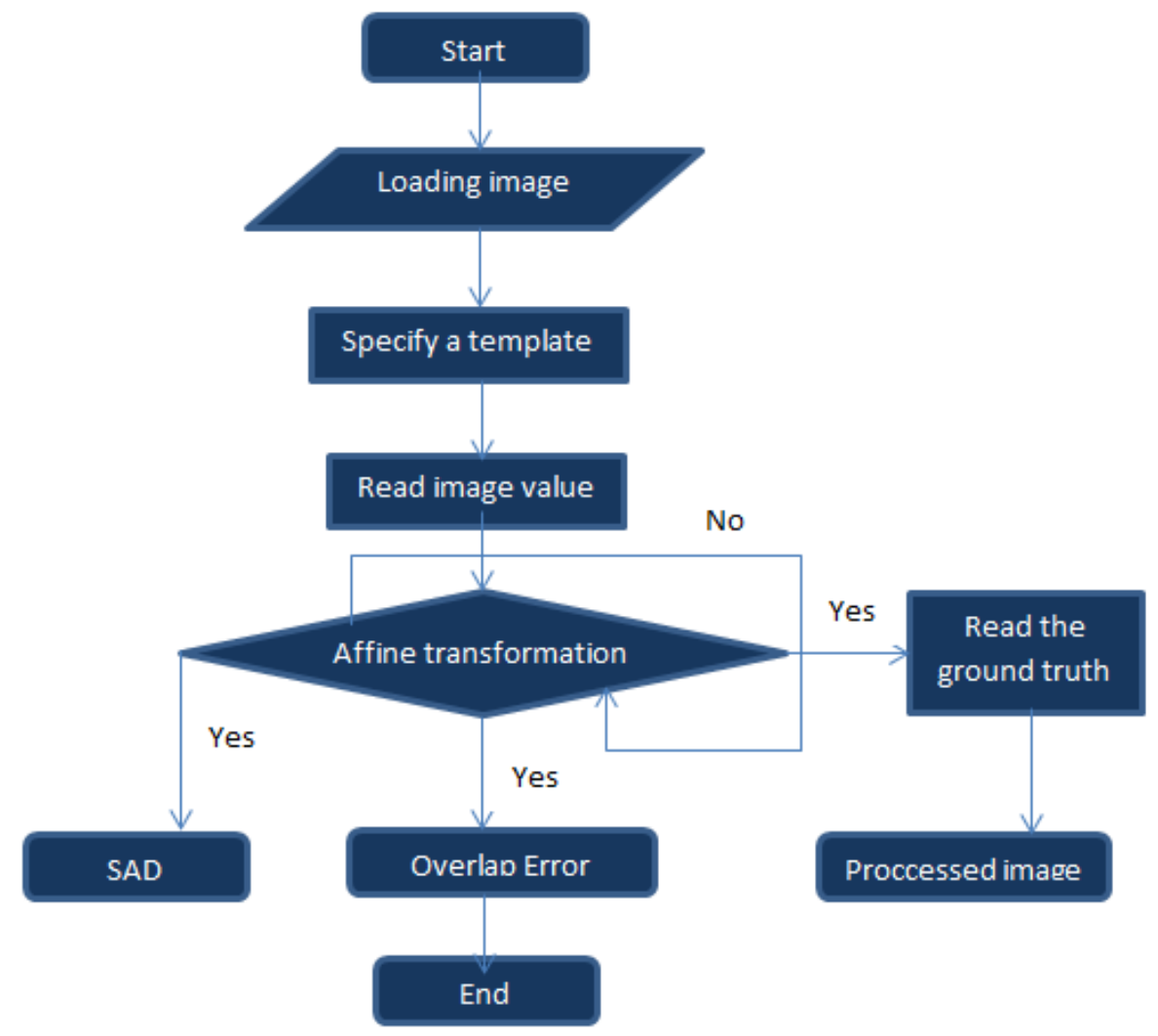

Figure 3. Research Stages 


\subsection{Testing}

With the aim of testing, this algorithm will be analyzed through the SAD value, the Overlap Error value, and the output in the form of a cut image. SAD (Sum of Absolute Difference Error) is a widely used tool to assess the similarity of two images. By comparing the matrix value of one of the pixels in the image, and continuing to move along the entire image[16] as in (1). Another testing has been done by overlap eror. This is one of the mapping steps to determine where the exact position of the template location on the image with ground and truth values is exactly in the area bounded by the green and magenta lines as shown in Figure 4.

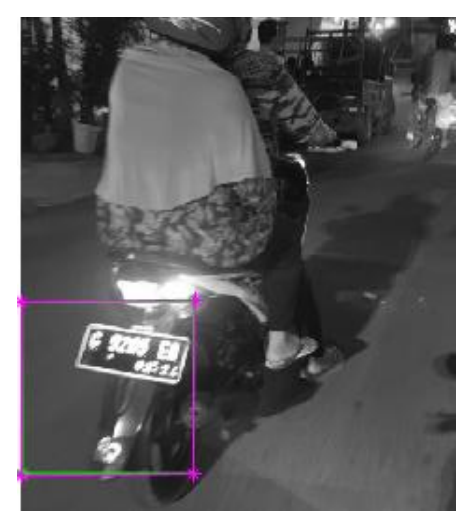

Figure 4. Illustration of Overlap Eror Position

\section{RESULTS AND DISCUSSION}

The image used in this research is a photo taken through a camera, with a visual image of a motorbike or car with a visible number plate, both from the front and from the side. There are 50 images, 12 of which are cars with front or rear parts, the remaining 38 are motorbikes with front, rear, or tilt image taking. Each image has a different resolution and there are several conditions such as low light and blur. Images have been named according to numerical order manually and in JPG format, in order to make sorting easier.

Following are the results of the analysis of the Fast Match algorithm with various conditions that can affect the measurement and results.

\subsection{Template Dimensions}

One of the sample images is the following image with a resolution of $1131 \times 747$, while the template made has a resolution of $374 \times 374$. The first step is to determine the dimensions of the template. Here is a comparison between the dimensions of the template as in Figure 4.

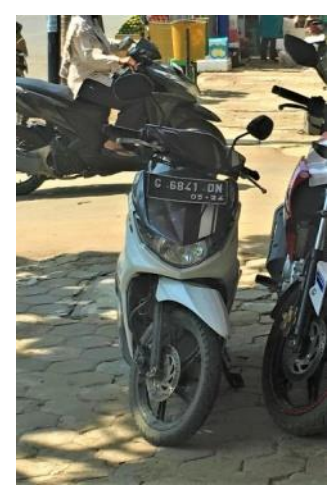

107 
Figure 4. Sample image tested

It can be seen from the table that the larger the size of the dimensions is the value of the smaller the template accelerates the processing time, and the resulting SAD value will be smaller due to the narrower image range as shown in Table 1.

Table 1. Dimensions Analysis

\begin{tabular}{|c|c|c|c|}
\hline & Resolution & Dimensions & SAD (Sum Of Absolute Difference) \\
\hline & $374 \times 374$ & 2 & 0.6 \\
\hline & $249 \times 249$ & 3 & 0.9 \\
\hline & & & \\
\hline & $150 \times 150$ & 5 & 0.9 \\
\hline
\end{tabular}

\subsection{Light Intesity}

Analysis of images with low light intensity and images that have high light intensity shown in Table 2.

Table 2. Light Intensity Analysis

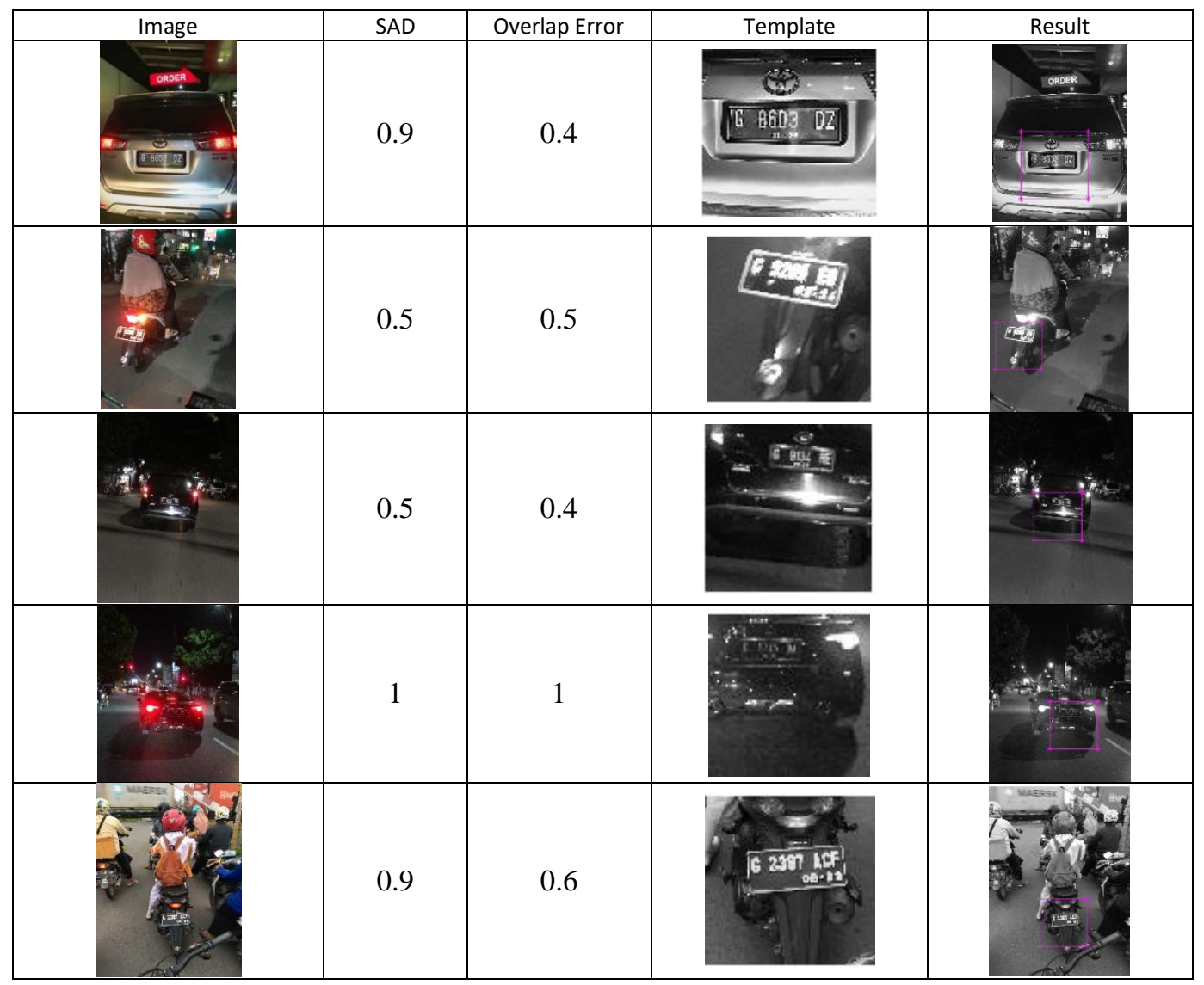




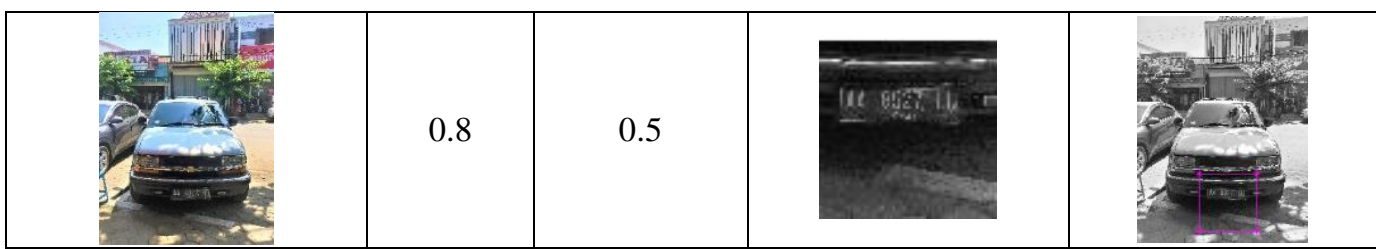

From the results of measurements that have been made, it can be seen that the level of similarity seen based on the SAD value is quite high in images that have low light intensity. Meanwhile, images that have high light intensity have a constant value of $0.3 \%$ of the predetermined template.

\section{CONCLUSION}

Based on a series of processes that have been carried out, several points had been raised. The geometry or positioning of the template greatly affects the SAD (Sum of Absolute Difference) and Overlap Error values where the wider the template is made, the more distance between the original image and the template, so that the similarity to the template image is very far away. The distance of the object being read affects the SAD value, compared to the close one, the distance of the object that is a little far away has a much better level of similarity to the template. Sufficient light intensity and not too high makes this algorithm work very well, as evidenced by the Overlap Error distance which is not too far from the template and the SAD value is not that big.

\section{REFERENCES}

[1] S. Saha, S. Basu, M. Nasipuri, and D. K. Basu, "A Hough Transform based Technique for Text Segmentation," J. Comput., vol. 2, no. 2, pp. 134-141, Feb. 2010.

[2] A. Mousa, "Canny Edge-Detection Based Vehicle Plate Recognition," Int. J. Signal Process. Image Process. Pattern Recognit., vol. 5, no. 3, pp. 1-8, 2012.

[3] I. Kusumadewi, C. A. Sari, D. R. I. Moses Setiadi, and E. H. Rachmawanto, "License Number Plate Recognition using Template Matching and Bounding Box Method," in Journal of Physics: Conference Series, 2019, vol. 1201, no. 1.

[4] M. M. Shidore and S. P. Narote, "Number Plate Recognition for Indian Vehicles," Acad. Res. Int., vol. 4, no. 3, pp. 48-55, 2013.

[5] F. Patel, J. Solanki, V. Rajguru, and A. Saxena, "Recognition of Vehicle Number Plate Using Image Processing Technique," Adv. Emerg. Med., vol. 7, no. 1, pp. 2-8, 2018.

[6] U. Dwivedi, P. Rajput, and M. K. Sharma, "License Plate Recognition System for Moving Vehicles Using Laplacian Edge Detector and Feature Extraction," Int. Res. J. Eng. Technol., vol. 4, no. 3, pp. 407-412, 2017.

[7] R. Panahi and I. Gholampour, "Accurate Detection and Recognition of Dirty Vehicle Plate Numbers for High-Speed Applications," IEEE Trans. Intell. Transp. Syst., vol. 18, no. 4, pp. 767-779, Apr. 2017.

[8] Z. Zhang and C. Wang, "The Research of Vehicle Plate Recognition Technical Based on BP Neural Network," AASRI Procedia, vol. 1, pp. 74-81, 2012.

[9] M. T. Qadri and M. Asif, "Automatic Number Plate Recognition System for Vehicle Identification Using Optical Character Recognition," in 2009 International Conference on Education Technology and Computer, 2009, pp. 335-338.

[10] S. Korman, D. Reichman, G. Tsur, and S. Avidan, "FasT-Match: Fast Affine Template Matching," in 2013 IEEE Conference on Computer Vision and Pattern Recognition, 2013, pp. 2331-2338. 
[11] J. Kim, J. K. Lee, and K. M. Lee, "Accurate Image Super-Resolution Using Very Deep Convolutional Networks," in 2016 IEEE Conference on Computer Vision and Pattern Recognition (CVPR), 2016, vol. 2016-Decem, pp. 1646-1654.

[12] S. Caraiman et al., "Computer Vision for the Visually Impaired: the Sound of Vision System," in 2017 IEEE International Conference on Computer Vision Workshops (ICCVW), 2017, vol. 2018-Janua, pp. 1480-1489.

[13] M. M. Azad, M. M. Hasan, and M. N. K, "Color Image Processing in Digital Image," Int. J. New Technol. Res., vol. 3, no. 3, pp. 56-62, 2017.

[14] X. Jun-bo, "Template matching algorithm based on gradient search," in 2014 International Conference on Mechatronics and Control (ICMC), 2014, no. Icmc, pp. 1472-1475.

[15] Y. M. Fouda, "A Robust Template Matching Algorithm Based on Reducing Dimensions," J. Signal Inf. Process., vol. 06, no. 02, pp. 109-122, 2015.

[16] H. Niitsuma and T. Maruyama, "Sum of Absolute Difference Implementations for Image Processing on FPGAs," in 2010 International Conference on Field Programmable Logic and Applications, 2010, no. D, pp. 167-170. 